\title{
EVALUATION OF GROWTH OF YOUNG COCONUT AND NUT YIELD OF OLD COCONUT AND THEIR NUTRIENT STATUS UNDER COCONUT-CASSAVA INTERCROPPING SYSTEMS
}

\author{
E. Andoh-Mensah, ${ }^{1}$ R. Nuhu Issaka ${ }^{2}$ and A. S. Ennin ${ }^{3}$ \\ ${ }^{1}$ CSIR - Oil Palm Research Institute, Coconut Programme, Box 245, Sekondi, Ghana. \\ ${ }^{1}$ e-mail: andomens@yahoo.com \\ ${ }^{2}$ CSIR - Soil Research Institute, Private Mail Bag, Academy Post Office, Kumasi, Ghana, \\ ${ }^{3}$ CSIR - Crops Research Institute, Box 3785, Kumasi, Ghana.
}

\begin{abstract}
Two on-farm experiments were carried out in the coconut belt of Southern Ghana from 2006 to 2009 to evaluate growth of young coconut plantings and nut yield of old coconut fields and their nutrient status under coconut-cassava intercropping systems. Experiment I was carried out in young MYD $x$ VTT coconut plantings. Experiment II was conducted in old West African Tall coconut plantings. The same cropping systems were evaluated under the two experiments in randomized complete block design with two replications each. The cropping systems were: 1. Sole coconut 2. Coconut + non-fertilized cassava 3. Coconut + cassava-fertilizer-I (Fertilizer I= 3045-45 kg/ha N-P $\left.\mathrm{O}_{5}-\mathrm{K}_{2} \mathrm{O}\right)$ and 4. Coconut + cassava-fertilizer-II (Fertilizer II = 60-45-90 kg/ ha $\left.\mathrm{N}-\mathrm{P}_{2} \mathrm{O}_{5}-\mathrm{K}_{2} \mathrm{O}\right)$. Young coconut planted as sole crop had significantly $(p<0.05)$ better growth than the intercropped. Coconut intercropped with non-fertilized cassava had superior growth relative to those intercropped with fertilized cassava. There was no significant $(p<0.05)$ difference in growth between coconut intercropped with cassava fertilizer I and those intercropped with cassava fertilizer II. Generally, the nutrient status of young coconut was not significantly (p>0.05) different between the cropping systems. Leaf $K$ was identified as a limiting factor to nut yield in the old coconut plots. Consequently, change in nut yield was closely linked to change in leaf $K$ with high coefficient of correlation $(r=0.798)$. Old coconut intercropped with fertilized cassava had significant $(P<0.05)$ increase in both leaf $K$ and nut yield while those intercropped with non-fertilized cassava suffered significant $(P<0.05)$ decline in both leaf $K$ and nut yield.
\end{abstract}

Keywords: Coconut-cassava intercropping, West African Tall, MYD x VTT, Leaf nutrients.

\section{INTRODUCTION}

The coconut palm, Cocos nucifera L., is undoubtedly an important cash crop in the economy of the coastal belt of Ghana (Adams et al., 1996). Unfortunately, the coconut industry is under a devastating threat by a lethal yellowing disease known in Ghana as Cape St. Paul Wilt Disease (Dery et al., 1997; 2008). Apart from the disease threat, the coconut industry is faced with the problem of low nut yield in the "disease-free zone" of the coconut belt due to poor weed control and soil fertility maintenance among others (Ofori and Nkansah-Poku, 1997).

The most practical way to ensure successful replanting of the devastated fields and to moti- 


\section{Andoh-Mensah et al.}

vate farmers to maintain old coconut farms is to develop suitable and profitable coconut-based cropping systems (Andoh-Mensah et al., 2005).

Intercropping is a major cropping system for coconut cultivation worldwide (Liyanage et al., 1985; Magat, 2004; Ohler, 2007). Intercropping represents a more efficient use of natural resources and labour (Fordham, 1983); broadens farmer's income/ food security base and helps in weed control (Bonneau and Sugarianto, 1999). Locally, a survey report indicated that 99\% of young coconut plantings in the coconut belt were intercropped with food crops and $79 \%$ of the intercrops involved cassava planting (Ollivier et al., 2006). Consequently, a study was initiated to develop appropriate coconutcassava intercropping systems to motivate farmers to replant their devastated fields and also maintain old coconut farms to improve nut yield. This paper evaluates growth of young coconut and nut yield of old coconut and their nutrient status under coconut-cassava intercropping systems.

\section{MATERIALS AND METHODS}

Two on-farm experiments were carried out in the Central and Western Regions in the coconut belt of Southern Ghana over a three-year period from 2006 to 2009.

\section{Experiment I}

Experiment I was carried out in young coconut plantings at two locations: Kusi and Antado in the Central Region of Ghana. Four cropping systems were evaluated in a randomized complete block design with two locational replications. The cropping systems were:

1. Sole coconut

2. Coconut+ non-fertilized cassava intercrop

3. Coconut + cassava-fertilizer-I intercrop (Fertilizer $\mathrm{I}=30-45-45 \mathrm{~kg} / \mathrm{ha} \mathrm{N}-\mathrm{P}_{2} \mathrm{O}_{5^{-}}$ $\mathrm{K}_{2} \mathrm{O}$ ) and 4. Coconut + cassava-fertilizerII intercrop (Fertilizer II $=60-45-90 \mathrm{~kg} / \mathrm{ha}$ $\mathrm{N}-\mathrm{P}_{2} \mathrm{O}_{5}-\mathrm{K}_{2} \mathrm{O}$ ).

The young coconut was established with Malayan Yellow Dwarf crossed to Vanuatu
Tall (MYD x VTT) hybrid known to have good agronomic characteristic and moderate tolerance to the Cape St. Paul Wilt Disease (Dery et al., 1997, 2005, 2008). Planting was done at $8.5 \mathrm{~m}$ triangular spacing with vertical inter-rows of $7.4 \mathrm{~m}$ wide. Plot size was $42.5 \mathrm{~m} \times 14.8 \mathrm{~m}$. A total of $48 \mathrm{~kg} \mathrm{~N} / \mathrm{ha} ; 62 \mathrm{~kg} \mathrm{P}_{2} \mathrm{O}_{5} / \mathrm{ha} ; 117 \mathrm{~kg} \mathrm{~K} 2 \mathrm{O} /$ ha and $38 \mathrm{~kg} \mathrm{MgO} /$ ha were applied in the form of urea, triple superphosphate (TSP); muriate of potash (MOP) and magnesium sulphate $\left(\mathrm{MgSO}_{4}\right)$ to the young coconut as basal fertilizers. Fertilizer dosage was split $44 \%$ and $56 \%$ and applied in years two and three in a ring form around coconut. Intercropping was done with an improved cassava variety ("CRIAgbelifia") one week after planting coconut and harvested 15 months later. Cassava was spaced $1 \mathrm{~m} \times 1 \mathrm{~m}$ in the vertical inter-rows but kept $1.7 \mathrm{~m}$ away from the coconut palms to obtain five rows of cassava between two rows of coconut. Fertilizer treatments in the form of Urea, TSP and MOP were split 50\% and applied 4 and 12 weeks after planting. The application was done in bands on two sides of the cassava stands and granules were buried in the soil.

Growth of MYD x VTT coconut hybrid was assessed by collar girth and leaf emission measurements at yearly interval. Collar girth was measured at the soil level. Coconut leaf rank four was sampled in year one and leaf rank nine was sampled in years two and three for analysis (Santos et al., 1996). GenStat Discovery Edition 3 statistical software was used for data analysis. Data were subjected to Two-Way Anova (in Randomized Blocks) and comparisons of interest $(\mathrm{C} 1, \mathrm{C} 2$ and $\mathrm{C} 3)$ were tested using ANOVA contrast. Comparisons with Fprobability $\leq 0.05$ were declared significant.

$\mathrm{C} 1$ : Sole coconut (CCsole) versus inter cropped coconut (CCic)

C2: Coconut with fertilized cassava intercrop (CC-CSV $\mathrm{F}_{\mathrm{F}}$ ) versus coconut with non- $\mathrm{fe} \mathrm{r}$ tilized cassava intercrop (CC-CSV $\mathrm{F}_{\mathrm{F}}$ )

C3: Coconut with cassava fertilizer I intercrop $\left(\mathrm{CC}-\mathrm{CSV}_{\mathrm{Fl}}\right)$ versus coconut with cassava fertilizer II intercrop (CC-CSV $\mathrm{Fll}_{\mathrm{Fl}}$ ). 


\section{Experiment II}

Experiment II was conducted in old coconut plantings at two locations: Menzezor and Nuba in the Western Region of Ghana. The old coconut trees aged 40-45 years were West African Tall (WAT) variety planted in a rectangular arrangement with average spacing of $7.7 \mathrm{~m}$ between rows and $8.8 \mathrm{~m}$ within rows. Plot size was $44.0 \mathrm{~m} \times 15.4 \mathrm{~m}$. The cropping systems evaluated and the design used was the same as in experiment I. Cassava intercropping and fertilization were also the same as in experiment I except that cassava was kept $1.85 \mathrm{~m}$ away from the old coconut. The old coconut trees were not fertilized. Coconut leaf sampling and analyses were done yearly. Leaf rank 14 was sampled. Nut yield was estimated annually by nut count of bunches subtended by leaf ranks 14, 19 and 24. The number of nuts in the three bunches were averaged and the mean multiplied by 12 to estimate nut load/ tree/ year (Santos et al., 1996). Data analyses were the same as in experiment I.

\section{RESULTS}

Experiment I

\section{Nutrient status of young coconut}

Leaf $\mathrm{Ca}$ of coconut intercropped with fertilized cassava (mean of $0.202 \%$ ) was significantly $(\mathrm{P}<0.05)$ lower than that of coconut intercropped with non-fertilized cassava (mean of $0.273 \%)$. There were no significant $(\mathrm{P}>0.05)$ differences between the cropping systems relative to leaf $\mathrm{N}, \mathrm{P}, \mathrm{K}$ and $\mathrm{Mg}$ even though leaf nutrient profile of sole coconut appeared to have advantage over intercropped coconut (Table 1).

Table 1: Variance and contrast analysis of leaf $\mathrm{N}, \mathrm{P}, \mathrm{K}, \mathrm{Mg}$ and Ca concentrations (\% dry wt) of young coconut hybrid in coconut-cassava intercropping systems

\begin{tabular}{|c|c|c|c|c|c|}
\hline Source of Variation & Df & $\begin{array}{l}\text { Sum of } \\
\text { Square }\end{array}$ & $\begin{array}{l}\text { Mean } \\
\text { Square }\end{array}$ & $\begin{array}{l}\text { Var. } \\
\text { Ratio }\end{array}$ & $\begin{array}{l}\text { F. } \\
\text { Prob. }\end{array}$ \\
\hline Cropping system: leaf $\mathbf{N}$ & 3 & 0.3404 & 0.1135 & 0.92 & $0.481^{\mathrm{ns}}$ \\
\hline \multicolumn{6}{|l|}{ Contrast } \\
\hline C1: CCsole (2.160\%) vs. CCic $(1.939 \%)$ & 1 & 0.1463 & 0.1463 & 1.18 & $0.313^{\mathrm{ns}}$ \\
\hline C2: CC-CSV ${ }_{\mathrm{F}}(1.958 \%) v s . \quad C C-\mathrm{CSV}_{\mathrm{F} 0}(1.903 \%)$ & 1 & 0.0081 & 0.0081 & 1.50 & $0.260^{\mathrm{ns}}$ \\
\hline C3: CC-CSV $\mathrm{Fl}_{\mathrm{Fl}}(1.805 \%)$ vs. CC-CSV $\mathrm{Fll}(2.110 \%)$ & 1 & 0.1860 & 0.1860 & 1.50 & $0.260^{\mathrm{ns}}$ \\
\hline Cropping system: leaf $\mathbf{P}$ & 3 & 0.0003 & 0.0001 & 0.43 & $0.740^{\mathrm{ns}}$ \\
\hline \multicolumn{6}{|l|}{ Contrast } \\
\hline C1: CCsole (0.145\%) vs. CCic $(0.137 \%)$ & 1 & 0.0002 & 0.0002 & 0.82 & $0.395^{\mathrm{ns}}$ \\
\hline C2: CC-CSV $_{\mathrm{F}}(0.139 \%)$ vs.CC-CSV $\mathrm{F} 0(0.133 \%)$ & 1 & 0.0001 & 0.0001 & 0.41 & $0.542^{\mathrm{ns}}$ \\
\hline C3: CC-CSV $V_{\mathrm{Fl}}(0.140 \%)$ vs. CC-CSV $\mathrm{Fll}(0.138 \%)$ & 1 & 0.0001 & 0.0001 & 0.05 & $0.831^{\mathrm{ns}}$ \\
\hline Cropping system: leaf $\mathbf{K}$ & 3 & 0.0394 & 0.0131 & 0.83 & $0.520^{\mathrm{ns}}$ \\
\hline \multicolumn{6}{|l|}{ Contrast } \\
\hline C1: CCsole $(0.843 \%)$ vs. CCic $(0.761 \%)$ & 1 & 0.0099 & 0.0099 & 0.62 & $0.455^{\mathrm{ns}}$ \\
\hline C2: $\mathrm{CC}_{-} \mathrm{CSV}_{\mathrm{F}}(0.761 \%)$ vs. CC-CSV $\mathrm{F}(0.843 \%)$ & 1 & 0.0150 & 0.0150 & 0.94 & $0.364^{\mathrm{ns}}$ \\
\hline C3: CC-CSV ${ }_{\mathrm{Fl}}(0.803 \%)$ vs. CC-CSV $\mathrm{Fll}(0.718 \%)$ & 1 & 0.0144 & 0.0144 & 0.91 & $0.372^{\mathrm{ns}}$ \\
\hline Cropping system: leaf Mg & 3 & 0.0051 & 0.0017 & 0.84 & $0.515^{\mathrm{ns}}$ \\
\hline \multicolumn{6}{|l|}{ Contrast } \\
\hline C1: CCsole (0.295\%) vs. CCic $(0.259 \%)$ & 1 & 0.0039 & 0.0039 & 1.91 & $0.210^{\mathrm{ns}}$ \\
\hline $\mathrm{C} 2: \mathrm{CC}-\mathrm{CSV}_{\mathrm{F}}(0.257 \%)$ vs. CC-CS $\mathrm{C} 0(0.265 \%)$ & 1 & 0.0002 & 0.0002 & 0.10 & $0.760^{\mathrm{ns}}$ \\
\hline C3: CC-CSV $V_{\mathrm{Fl}}(0.268 \%)$ vs. CC-CSV $\mathrm{Fll}(0.245 \%)$ & 1 & 0.0010 & 0.0010 & 0.50 & $0.502^{\mathrm{ns}}$ \\
\hline Cropping system: leaf Ca & 3 & 0.0205 & 0.0068 & 3.36 & $0.084^{\mathrm{ns}}$ \\
\hline \multicolumn{6}{|l|}{ Contrast } \\
\hline C1: CCsole $(0.183 \%)$ vs. CCic $(0.225 \%)$ & 1 & 0.0054 & 0.0054 & 2.67 & $0.146^{\mathrm{ns}}$ \\
\hline C2: CC-CSV $\mathrm{F}(0.202 \%)$ vs. CC-CSV $\mathrm{F} 0(0.273 \%)$ & 1 & 0.0135 & 0.0135 & 6.68 & $0.036 *$ \\
\hline C3: CC-CSV $\mathrm{Fl}_{\mathrm{Fl}}(0.215 \%)$ vs.CC-CSV $\mathrm{Fll}(0.188 \%)$ & 1 & 0.0015 & 0.0015 & 0.75 & $0.416^{\mathrm{ns}}$ \\
\hline *Significant at $P \leq 0.05$ & & nificont & 0.05 & & ersus \\
\hline
\end{tabular}




\section{Growth of young coconut}

Growth of young coconut as measured by collar girth and cumulative leaf number was significantly $(\mathrm{P} \leq 0.01)$ affected by coconut-cassava intercropping systems. Contrast analysis of collar girth (Table 2) indicates a significant $(\mathrm{P}<0.01)$ difference between sole coconut (mean girth of $53.30 \mathrm{~cm}$ ) and intercropped coconut (mean girth of $43.80 \mathrm{~cm}$ ). Collar girth of coconut intercropped with fertilized cassava (mean of $42.05 \mathrm{~cm})$ was significantly $(\mathrm{P}<0.05)$ lower than collar girth of those intercropped with non-fertilized cassava (mean of $47.31 \mathrm{~cm}$ ). There was no significant difference $(\mathrm{P}>0.05)$ in collar girth between coconut intercropped with cassava fertilizer I (mean girth of $42.74 \mathrm{~cm}$ ) and those intercropped with cassava fertilizer II (mean girth of $41.35 \mathrm{~cm}$ ).

Contrast analysis of cumulative leaf number (Table 3) also shows a significant $(\mathrm{P}<0.01)$ difference between sole coconut (mean leaf number of 15.8) and intercropped coconut (mean leaf number of 14.7). There was no significant difference $(\mathrm{P}>0.05)$ in cumulative leaf number between coconut intercropped with fertilized cassava (mean leaf number of 14.7) and those intercropped with non-fertilized cassava (mean leaf number of 14.9). Similarly, there was no significant difference $(\mathrm{P}>0.05)$ in cumulative leaf number between coconuts intercropped with cassava fertilizer I (mean leaf number of 15.0) and those intercropped with cassava fertilizer II (mean of leaf number of 14.4).

Growth parameters increased significantly $(\mathrm{P}<0.01)$ across the sampling times for all the cropping systems. However, the interaction effects between cropping system and time on the growth parameters were not significant $(\mathrm{P}>0.05)$.

\section{Experiment II}

Leaf nutrient status of old coconut

Apart from leaf K, nutrient profiles of old

Table 2: Variance and contrast analysis of collar girth $(\mathrm{cm})$ of young coconut hybrid in coconut-cassava intercropping systems

\begin{tabular}{|c|c|c|c|c|c|}
\hline Source of Variation & Df & $\begin{array}{l}\text { Sum of } \\
\text { Square }\end{array}$ & $\begin{array}{l}\text { Mean } \\
\text { Square }\end{array}$ & $\begin{array}{l}\text { Var. } \\
\text { Ratio }\end{array}$ & $\begin{array}{l}\text { F. } \\
\text { Prob. }\end{array}$ \\
\hline Location & 1 & 114.76 & 114.76 & 3.76 & - \\
\hline Cropping system & 3 & 697.25 & 232.42 & 7.62 & $<0.01 * *$ \\
\hline \multicolumn{6}{|l|}{ Contrast } \\
\hline C1: CCsole $(53.30 \mathrm{~cm})$ vs. CCic $(43.80 \mathrm{~cm})$ & 1 & 541.50 & 541.50 & 17.76 & $<0.01 * *$ \\
\hline $\begin{array}{l}\text { C2: CC-CSV } \mathrm{F}(42.0 \mathrm{~cm}) v s . \text { CC-CSV } \\
(47.31 \mathrm{~cm})\end{array}$ & 1 & 148.05 & 148.05 & 4.86 & $0.044 *$ \\
\hline $\begin{array}{l}\text { C3: CC-CSV } \mathrm{Fl}_{\mathrm{Fl}}(42.74 \mathrm{~cm}) \text { vs. CC-CSV } \mathrm{Fll} \\
(41.35 \mathrm{~cm})\end{array}$ & 1 & 7.70 & 7.70 & 0.25 & $0.623^{\mathrm{ns}}$ \\
\hline Time & 3 & 28092.71 & 9364.24 & 307.1 & $<0.01 * *$ \\
\hline Cropping system .Time & 9 & 507.62 & 56.40 & 1.85 & $0.140^{\mathrm{ns}}$ \\
\hline Contrast & & & & & \\
\hline C1: CCsole vs. CCic & 3 & 245.18 & 81.73 & 2.68 & $0.084^{\mathrm{ns}}$ \\
\hline $\mathrm{C} 2: \mathrm{CC}-\mathrm{CSV}_{\mathrm{F}}$ vs. CC-CSV $\mathrm{F} 0$ & 3 & 252.63 & 84.21 & 2.76 & $0.078^{\mathrm{ns}}$ \\
\hline C3: CC-CSV $\mathrm{Fl}_{\text {l }}$ s. CC-CSV & 3 & 9.81 & 3.27 & 0.11 & $0.955^{\mathrm{ns}}$ \\
\hline Residual & 15 & 457.40 & 30.49 & - & \\
\hline Total & 31 & 29869.74 & - & - & \\
\hline
\end{tabular}

*Significant at $P<0.05 \quad * *$ Significant at $P<0.01 \quad{ }^{n s}$ Not Significant at $P=0.05 \quad v s=$ Versus 
Evaluation of growth of young coconut...

Table 3: Variance and contrast analysis of cumulative leaf number (Ln) of young coconut hybrid in coconut-cassava intercropping systems

\begin{tabular}{|c|c|c|c|c|c|}
\hline \multicolumn{6}{|c|}{ Source of variation } \\
\hline \multicolumn{6}{|l|}{ Location } \\
\hline \multicolumn{6}{|c|}{ Cropping System } \\
\hline \multicolumn{6}{|l|}{ Contrast } \\
\hline \multicolumn{6}{|c|}{ C1: CCsole (Ln 15.8 ) vs. CCic (Ln 14.7) } \\
\hline \multicolumn{6}{|c|}{$\mathrm{C} 2: \mathrm{CC}-\mathrm{CSV}_{\mathrm{F}}(\operatorname{Ln} 14.7)$ vs. CC-CSV $\mathrm{F0}(\operatorname{Ln} 14.9)$} \\
\hline \multicolumn{6}{|c|}{$\mathrm{C} 3: \mathrm{CC}^{-\mathrm{CSV}_{\mathrm{Fl}}}(\operatorname{Ln} 15.0)$ vs. CC-CSV $\mathrm{Fll}(\operatorname{Ln} 14.4)$} \\
\hline \multicolumn{6}{|l|}{ Time } \\
\hline \multicolumn{6}{|c|}{ Cropping system .Time } \\
\hline \multicolumn{6}{|c|}{ Contrast } \\
\hline \multicolumn{6}{|c|}{ C1:CCsole vs. CCic } \\
\hline \multicolumn{6}{|c|}{ C2: CC-CSV $V_{\mathrm{F}} v s . \mathrm{CC}-\mathrm{CSV}_{\mathrm{F} 0}$} \\
\hline \multicolumn{6}{|c|}{ C3: CC-CSV $V_{\mathrm{Fl}}$ vs. CC-CSV Fll } \\
\hline \multicolumn{6}{|l|}{ Residual } \\
\hline \multicolumn{6}{|l|}{ Total } \\
\hline \multicolumn{6}{|c|}{ *Significant at $P<0.05$} \\
\hline \multicolumn{6}{|c|}{$\begin{array}{l}\text { WAT trees at the onset of the study generally } \\
\text { met the critical levels for optimum nut yield } \\
\text { (Table 4). }\end{array}$} \\
\hline \multicolumn{6}{|c|}{$\begin{array}{l}\text { Table 4: Percent leaf } \mathrm{N}, \mathrm{P}, \mathrm{K}, \mathrm{Mg} \text { and } \mathrm{Ca} \\
\text { concentrations of old WAT trees in coconut- } \\
\text { cassava intercropping systems at the onset of } \\
\text { trial }\end{array}$} \\
\hline $\begin{array}{l}\text { Cropping } \\
\text { system }\end{array}$ & $1 \mathrm{v}$ & $\mathbf{P}$ & $\begin{array}{l}6 \text { dry } \\
\text { K }\end{array}$ & 1719 & \\
\hline CCsole & 1.82 & 0.14 & 0.47 & 0.18 & 0.31 \\
\hline $\mathrm{CC}-\mathrm{CSV}_{\mathrm{F} 0}$ & 1.82 & 0.17 & 0.64 & 0.24 & 0.35 \\
\hline $\mathrm{CC}-\mathrm{CSV} \mathrm{Fl}_{\mathrm{Fl}}$ & 1.84 & 0.12 & 0.47 & 0.27 & 0.30 \\
\hline CC-CSV & 1.80 & 0.12 & 0.59 & 0.30 & 0.33 \\
\hline $\mathrm{CC}-\mathrm{CSV}_{\mathrm{F}}$ & 1.82 & 0.12 & 0.53 & 0.29 & 0.32 \\
\hline $\mathrm{CCic}$ & 1.82 & 0.13 & 0.62 & 0.28 & 0.33 \\
\hline $\begin{array}{l}{ }^{4} \text { Critical } \\
\text { levels }\end{array}$ & 1.80 & 0.12 & 0.80 & 0.20 & 0.32 \\
\hline
\end{tabular}

${ }^{4}$ Magat, 2003

Percent change in nutrient profile over the study period was significant $(\mathrm{p}<0.05)$ for leaf $\mathrm{K}$ and $\mathrm{Mg}$ but not leaf $\mathrm{N}, \mathrm{P}$ and $\mathrm{Ca}$ (Table 5). Contrast analysis of leaf $\mathrm{K}$ indicates a signifi-

\begin{tabular}{lllll} 
Df & $\begin{array}{l}\text { Sum of } \\
\text { Square }\end{array}$ & $\begin{array}{l}\text { Mean } \\
\text { Square }\end{array}$ & $\begin{array}{l}\text { Var. } \\
\text { Ratio }\end{array}$ & $\begin{array}{l}\text { F. } \\
\text { Prob. }\end{array}$ \\
\hline 1 & 4.50 & 4.50 & 7.53 & - \\
3 & 8.92 & 2.97 & 4.97 & $0.01^{* *}$
\end{tabular}

\begin{tabular}{lllll}
1 & 7.15 & 7.15 & 11.96 & $<0.01^{* *}$ \\
1 & 0.33 & 0.33 & 0.56 & $0.467^{\mathrm{ns}}$ \\
1 & 1.44 & 1.44 & 2.41 & $0.142^{\mathrm{ns}}$ \\
3 & 2026.83 & 675.61 & 1126.02 & $<0.01^{* *}$ \\
9 & 4.52 & 0.50 & 0.84 & $0.592^{\mathrm{ns}}$ \\
3 & 3.87 & 1.29 & 2.16 & $0.135^{\mathrm{ns}}$ \\
3 & 0.51 & 0.17 & 0.29 & $0.835^{\mathrm{ns}}$ \\
3 & 0.14 & 0.05 & 0.08 & $0.972^{\mathrm{ns}}$ \\
15 & 8.97 & 0.60 & - & \\
31 & 2053.75 & - & - & \\
\hline
\end{tabular}

${ }^{n s}$ Not Significant at $P=0.05 \quad v s=$ Versus

cant $(\mathrm{P}=0.01)$ difference between coconut intercropped with fertilized cassava (change in $\% \mathrm{~K}, 0.190$ ) and coconut intercropped with non -fertilized cassava (change in \% K, -0.200). For leaf $\mathrm{Mg}$, contrast analysis shows a significant $(\mathrm{P}<0.05)$ difference between sole coconut (change in \% Mg, 0.185) and intercropped coconut (change in \% Mg, 0.125).

\section{Nut gain in old coconut}

Nut yield of old coconut was significantly $(\mathrm{P}<0.05)$ affected by coconut-cassava intercropping systems. Contrast analysis of nut gain over the study period (Table 6) indicates a highly significant $(\mathrm{P}<0.01)$ difference between coconut intercropped with fertilized cassava (nut gain of 2,372/ha) and coconut intercropped with non-fertilized cassava (nut gain of $-1,228$ / ha).

Nut gain by intercropped coconut (1,172 nuts/ ha) appeared superior to that of sole coconut (480 nuts/ha). Similarly, nut gain by coconut intercropped with cassava fertilizer I $(3,064$ 
Table 5: Variance and contrast analysis of change in leaf $\mathrm{N}, \mathrm{P}, \mathrm{K}, \mathrm{Mg}$ and Ca concentrations (\% dry wt) of old WAT trees in coconut-cassava intercropping systems

\begin{tabular}{|c|c|c|c|c|c|}
\hline Source of variation & Df & $\begin{array}{l}\text { Sum of } \\
\text { Square }\end{array}$ & $\begin{array}{l}\text { Mean } \\
\text { Square }\end{array}$ & $\begin{array}{l}\text { Var. } \\
\text { Ratio }\end{array}$ & $\begin{array}{l}\text { F. } \\
\text { Prob. }\end{array}$ \\
\hline Cropping system: $\Delta$ leaf $\mathrm{N}$ & 3 & 0.0870 & 0.0290 & 0.79 & $0.574^{\mathrm{ns}}$ \\
\hline \multicolumn{6}{|l|}{ Contrast } \\
\hline C1: CCsole $(-0.005 \%)$ vs. CCic $(0.137 \%)$ & 1 & 0.0301 & 0.0301 & 0.82 & $0.432^{\mathrm{ns}}$ \\
\hline 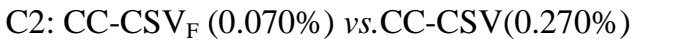 & 1 & 0.0533 & 0.0533 & 1.45 & $0.314^{\mathrm{ns}}$ \\
\hline C3: CC-CSV $\mathrm{Fl}(0.100 \%)$ vs. CC-CSV $\mathrm{Fl}_{\mathrm{Fl}}(0.040 \%)$ & 1 & 0.0036 & 0.0036 & 0.10 & $0.775^{\mathrm{ns}}$ \\
\hline Cropping system: $\Delta$ leaf $P$ & 3 & 0.0017 & 0.0006 & 0.34 & $0.801^{\mathrm{ns}}$ \\
\hline \multicolumn{6}{|l|}{ Contrast } \\
\hline C1: CCsole $(-0.005 \%)$ vs. CCic $(-0.007 \%)$ & 1 & 0.00004 & 0.00004 & 0.00 & $0.964^{\mathrm{ns}}$ \\
\hline C2: CC-CSV $(0.005 \%) v s . C C-\mathrm{CSV}_{\mathrm{F} 0}(0.030 \%)$ & 1 & 0.0016 & 0.0016 & 0.95 & $0.401^{\mathrm{ns}}$ \\
\hline C3: CC-CSV $\mathrm{Fl}(0.000 \%)$ vs. CC-CSV $\mathrm{Fl}_{\mathrm{Fl}}(0.010 \%)$ & 1 & 0.0001 & 0.0001 & 0.06 & $0.825^{\mathrm{ns}}$ \\
\hline Cropping system: $\Delta$ leaf $K$ & 3 & 0.2052 & 0.0684 & 11.93 & $0.036^{*}$ \\
\hline \multicolumn{6}{|l|}{ Contrast } \\
\hline C1: CCsole $(0.110 \%)$ vs. CCic $(0.180 \%)$ & 1 & 0.0024 & 0.0024 & 0.420 & $0.564^{\mathrm{ns}}$ \\
\hline C2: CC-CSV $_{\mathrm{F}}(0.190 \%) v s . \mathrm{CC}-\mathrm{CSVV}_{\mathrm{F}}(0.200 \%)$ & 1 & 0.2028 & 0.2028 & 35.37 & $0.01 * *$ \\
\hline C3: CC-CSV $\mathrm{Fl}(0.190 \%)$ vs. CC-CSV $\mathrm{FII}(0.190 \%)$ & 1 & 0.0000 & 0.0000 & 0.000 & $1.000^{\mathrm{ns}}$ \\
\hline Cropping system: $\Delta$ leaf $\mathrm{Mg}$ & 3 & 0.0383 & 0.0128 & 9.81 & $<0.046^{*}$ \\
\hline \multicolumn{6}{|l|}{ Contrast } \\
\hline C1: CCsole $(0.185 \%)$ vs. CCic $(0.125 \%)$ & 1 & 0.0308 & 0.0308 & 23.71 & $<0.017 *$ \\
\hline 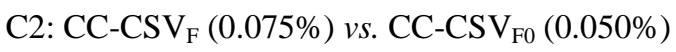 & 1 & 0.00002 & 0.0002 & 0.16 & $0.716^{\mathrm{ns}}$ \\
\hline C3: CC-CSV $-\mathrm{Fl}(0.080 \%)$ vs. CC-CSV $\mathrm{FII}(0.005 \%)$ & 1 & 0.0072 & 0.0072 & 5.56 & $0.100^{\mathrm{ns}}$ \\
\hline Cropping system: $\Delta$ leaf $\mathrm{Ca}$ & 3 & 0.0077 & 0.0026 & 1.89 & $0.307^{\mathrm{ns}}$ \\
\hline \multicolumn{6}{|l|}{ Contrast } \\
\hline C1: CCsole $(0.055 \%)$ vs. CCic $(0.072 \%)$ & 1 & 0.0004 & 0.0004 & 0.31 & $0.617^{\mathrm{ns}}$ \\
\hline C2: CC-CSV $(0.093 \%) v s$. CC-CSV ${ }_{F 0}(0.030 \%)$ & 1 & 0.0052 & 0.0052 & 3.86 & $0.144^{\mathrm{ns}}$ \\
\hline C3: $\mathrm{CC}^{-\mathrm{CSVV}_{\mathrm{Fl}}}(0.115 \%)$ vs. CC-CSV $\mathrm{FII}(0.070 \%)$ & 1 & 0.0020 & 0.0020 & 1.50 & $0.308^{\mathrm{ns}}$ \\
\hline
\end{tabular}

*Significant at $P \leq 0.05 * *$ Sig. at $P \leq 0.01{ }^{n s}$ Not Significant at $P=0.05 \quad \Delta=$ Change from initial to final

nuts/ha) looked superior to that of coconut intercropped with cassava fertilizer II $(1,680$ nuts/ha). The huge differences however were not statistically significant $(\mathrm{P}>0.05)$. Nut gain varied significantly $(\mathrm{P}<0.01)$ across sampling times. However, interaction effect between cropping system and time on nut gain was not significant $(\mathrm{P}>0.05)$.

\section{DISCUSSION}

\section{Experiment I}

Growth of young MYD x VTT coconut hybrid did not suffer stagnation under the cropping systems as increases in collar girth and cumula- tive leaf number across the sampling times were significant $(\mathrm{P}<0.05)$. The reduced vigor observed in the growth of intercropped coconut relative to sole coconut may be attributed to interspecific competition between coconut and cassava. According to Begon et al. (2006) interspecific competition occurs when individuals of two separate species share a limiting resource in the same location leading to lowered fecundity, growth or survival in at least one species.

In this instance, it was the coconut hybrid which suffered lowered growth; probably 
Evaluation of growth of young coconut...

Table 6: Variance and contrast analysis of nut gain per ha of old WAT trees in coconutcassava intercropping systems

\begin{tabular}{|c|c|c|c|c|c|}
\hline Source of variation & Df & $\begin{array}{l}\text { Sum of } \\
\text { Square }\end{array}$ & $\begin{array}{l}\text { Mean } \\
\text { Square }\end{array}$ & $\begin{array}{l}\text { Var. } \\
\text { Ratio }\end{array}$ & $\begin{array}{l}\text { F. } \\
\text { Prob. }\end{array}$ \\
\hline Location & 1 & 1106704 & 1106704 & 0.42 & - \\
\hline Cropping System & 3 & 46578224 & 15526075 & 5.83 & $0.026 *$ \\
\hline \multicolumn{6}{|l|}{ Contrast } \\
\hline C1: CCsole (-480 nuts/ha) vs. CCic (1,172 nuts/ha) & 1 & 8187312 & 8187312 & 3.07 & $0.123^{\mathrm{ns}}$ \\
\hline $\begin{array}{l}\text { C2: CC-CSV } \\
1,228 \text { nuts/ ha })\end{array}$ & 1 & 34560000 & 34560000 & 12.98 & $\begin{array}{l}<0.01 * \\
*\end{array}$ \\
\hline $\begin{array}{l}\text { C3: CC-CSV } \\
\text { (1,680 nuts/ ha) }\end{array}$ & 1 & 3830912 & 3830912 & 1.44 & $0.269^{\mathrm{ns}}$ \\
\hline Time & 1 & 16679056 & 16679056 & 6.26 & $0.041 *$ \\
\hline $\begin{array}{l}\text { Cropping system .Time } \\
\text { Contrast }\end{array}$ & 3 & 1287600 & 429200 & 0.16 & $0.919^{\mathrm{ns}}$ \\
\hline C1: CCsole vs. CCic & 1 & 170885 & 170885 & 0.06 & $0.807^{\mathrm{ns}}$ \\
\hline $\mathrm{C} 2: \mathrm{CC}^{-} \mathrm{CSV}_{\mathrm{F}}$ vs. CC-CSV $\mathrm{F}_{\mathrm{F}}$ & 1 & 624683 & 624683 & 0.23 & $0.643^{\mathrm{ns}}$ \\
\hline $\mathrm{C} 3: \mathrm{CC}-\mathrm{CSV}_{\mathrm{Fl}}$ vs. CC-CSV Fll & 1 & 492032 & 492032 & 0.18 & $0.680^{\mathrm{ns}}$ \\
\hline Residual & 7 & 18643056 & 2663294 & - & - \\
\hline Total & 15 & 84294640 & - & - & - \\
\hline
\end{tabular}

*Significant at $P<0.05 \quad * *$ Significant at $P<0.01$

${ }^{n s}$ Not Significant at $P=0.05 \quad v s=$ Versus

through reduced nutrient uptake due to the aggressive rooting system of cassava (FAO, 2010). Consequently, the observed nutrient status of the intercropped coconut especially leaf $\mathrm{N}, \mathrm{P}, \mathrm{K}$ and $\mathrm{Mg}$ concentrations appeared less superior to that of the sole coconut. The competition probably grew keener with fertilization of cassava as it triggered more active growth and tuberization (Ennin et al., 2009); leading to enhanced competitive ability in favor of cassava. This probably explains the superior growth and nutrient status particularly leaf $\mathrm{Ca}$, $\mathrm{Mg}$ and $\mathrm{K}$ observed in young coconut hybrid intercropped with non-fertilized cassava relative to those intercropped with fertilized cassava.

\section{Experiment II}

Leaf $\mathrm{K}$ ranging from $0.47-0.64 \%$ at the onset of the study in the old coconut (Table 4) was a major limiting factor to nut yield given a critical leaf $\mathrm{K}$ value of $0.8 \%$ for optimum nut yield (Magat, 2003). Consequently, change in nut yield of old coconut in the cropping systems was closely linked to change in leaf $\mathrm{K}$ with significant $(\mathrm{p}<0.05)$ coefficient of correlation $(\mathrm{r}$ $=0.798)$. This is consistent with the observation made by Andoh-Mensah et al. (2003) in which nut yield response to fertilizer application in old coconut was dependent on the level of improvement in the limiting $\mathrm{P}$.

Change in leaf $\mathrm{K}$ was driven by the cropping systems. Old coconut intercropped with fertilized cassava benefited from the residual effect of cassava fertilization (Andoh-Mensah et al., 2005) leading to significant $(\mathrm{P}<0.05)$ increase in leaf $\mathrm{K}$ and nut yield. The reverse was true for old coconut intercropped with non-fertilized cassava which suffered significant $(\mathrm{P}<0.05)$ 
decline in leaf $\mathrm{K}$ and nut yield. Though leaf $\mathrm{Mg}$ increased significantly $(\mathrm{P}<0.05)$ in sole coconut, it did not impact on nut yield since it was not a limiting factor.

\section{ACKNOWLEDEMENT}

The authors duly acknowledge the financial assistance given by the Ghana - French Technical Cooperation through the FSP project. They also wish to express their gratitude to the following staff of Ministry of Food and Agriculture: Messrs Y. Mustapha of KEEA, M. Asare of Ahanta West, P. I. Danquah of Jomoro, C. Anane and D Asihene of Nzema East for their immense field assistance. The technical support offered in the area of data collection and treatment application by Mr. S. Obeng Antwi of the Crops Research Institute and Messrs. Richard Nkrumah, Kenneth Atta-Peters and Martin Bannerman of OPRI Coconut Research Programme are duly recognized.

\section{REFERENCES}

Adams, M., Arthur, R., Duhamel, G., Ghartey, N., Overfield, D., and Willougby, N. (1996). Analysis of natural resource utilization, livelihoods systems coping strategies and natural resource research needs in coconut growing areas of the coastal zone ecosystems in Ghana. NRI DOC CO834, Vol. 1, Main report.

Andoh-Mensah, E., Bonneau, X., Nuertey, B. N. and Dery, S. K. (2003). Effect of mineral nutrition on nut yield and fruit composition of ma ture coconut palms in the coastal belt of Western Region of Ghana: Preliminary Studies. CORD XIX (2): 11-19.

Andoh-Mensah, E., Bonneau, X., Ollivier, J., Nuertey, B. N. and Dery, S. K. (2005). Evaluation of crop management options for replanting of coconut plantations devastated by lethal yellowing disease in Ghana. CORD 21 (2): 63-67.

Begon, M., Townsend, C. R. and Harper, J. L.
(2006). Ecology: From_individuals to ecosystems. $4^{\text {th }}$ edition, Blackwell Publishers, Malden, MA.

Bonneau, X. and Sugarianto, J. (1999). Intercropping with young hybrid coconut palms in climatically marginal areas. Plant. Rech. Dévelop. 6(1):13-29

Dery, S. K., N'cho, Y. P., Sangaré A. and Arkhurst E. D. (1997). Cape St. Paul Wilt Disease: Resistance screening and prospects for rehabilitating the coconut industry in Ghana. In: Eden Green, S. J. and Ofori, F. (Editors). Proceedings of international workshop on lethal yellow ing-like disease of coconut, Elmina, Ghana. NRI, Chatman, UK, pp.147-151

Dery, S. K., Owusu-Nipah, J., Andoh-Mensah, E., Nuertey, B. N., Nkansah-Poku, J., Arthur, R. and Philippe, R. (2005). On-farm evaluation of the coconut hybrid, Malayan Yellow Dwarf x Vanuatu Tall for tolerance to the lethal yellowing disease of coconut in Ghana. CORD 21 (1): 50-56.

Dery, S. K., Philippe, R., Baudouin, L., Quai coe, R. N., Nkansah-Poku J., OwusuNipah J., Arthur R., Dare, D., Yankey, N. and Dollet, M. (2008). Genetic diversity among coconut varieties for susceptibility to CSPWD. Euphytica 164:1-11

Ennin, A. S., Banful, B., Andoh-Mensah, E., Nuhu Issaka, R., Nii Lamptey, J., Aduening-Manu, J., Bolfrey-Arku, G. and Dery, S. K. (2009). Food crop intercropping alternatives for replanting coconut farms destroyed by lethal yellowing disease. Journal of Food, Agriculture \& Environment, 7(3\&4): 581-587.

FAO, (2010). Home garden technology leaflet 12. FAO Corporate Documentary Repository. http://www.fao.org/ D O CREP/003/X3996E/x3996e36.htm 
Fordham, R. (1983). Intercropping: what are the advantages? Outlook on Agriculture 12(3):142-146

Liyanage, M., Tejwani, S. K. G. and Nair, P. K. R. (1985). Intercropping under coconut in Sri Lanka. Agroforestry Systems 2:215-228.

Magat, S. S. (2003). Coconut leaf nutrient levels of bearing dwarf varieties and physiological, critical and adequacy levels in crop nutrition management. Cord XIX (2): $1-10$.

Magat, S. S. (2004). Growing of intercrops in coconut lads to generate more food and agricultural products, jobs and enhancing farm incomes. Coconut intercropping Salient notes. Dept. of Agric., Phillippine Coconut Authority, Quezon City, Philip pines, pp. 7

Ofori, F. and Nkansah-Poku, J. (1997). Cape St. Paul Wilt Disease of Coconut in Ghana: History of its occurrence and spread. In: Eden Green, S. J. and Ofori, F. (Editors). Proceedings of International Workshop on Lethal Yellowing-Like Disease of Coconut, Elmina, Ghana. NRI, Chatman, UK, pp. 27-32

Ohler, J. G. (2007). Moder n coconut man agement; palm cultivation and products. FAO, Rome. http://ecoport.org/ep? Sear $\underline{\text { chType }=\text { earticleView\&earticleld }=127 \& p a}$ ge $=1383$

Ollivier, J., Andoh-Mensah, E., Ransford, A. and Dery, S. K. (2006). Farmers' strategies and replanting performance in the context of coconut lethal yellowing disease along the coastal region in Ghana. CORD 22 (1): 66-75.

Santos, G. A., Batugal, P. A., Othman, A., Baudouin, L. and Laboisse, J. P. (Editors). (1996). Manual on standardized research techniques in coconut breeding. IPGRI- APO, Serdang, Malaysia. 